\title{
Determination of the material properties of polymers using laser-generated broadband ultrasound
}

\author{
Leander Claes, Thorsten Meyer, Fabian Bause, Jens Rautenberg, and Bernd Henning \\ Measurement Engineering Group, Paderborn University, Warburger Str. 100, 33098 Paderborn, Germany \\ Correspondence to: Leander Claes (claes@emt.uni-paderborn.de)
}

Received: 23 September 2015 - Revised: 19 May 2016 - Accepted: 22 May 2016 - Published: 7 June 2016

\begin{abstract}
In the non-destructive determination of material properties, the utilization of ultrasound has proven to be a viable tool. In the presented paper, a laser is used to create broadband acoustic waves in plate-shaped specimens by applying the photoacoustic effect. The waves are detected using a purpose-built ultrasonic transducer that is based on piezoceramics instead of the commonly used piezoelectric polymer films. This new transducer concept allows for detection of ultrasonic waves up to $10 \mathrm{MHz}$ with high sensitivity, thereby allowing the characterization of highly damping materials such as polymers. The recorded data are analysed using different methods to obtain information on the propagation modes transmitted along the specimen. In an inverse procedure, the gained results are compared to simulations, yielding approximations for the specimen's material properties.
\end{abstract}

\section{Introduction}

Ultrasound transmission measurements are often used for non-destructive testing and material characterization. In contrast to destructive testing and characterization methods, no reference sample is required. Instead, individual components can be tested (multiple times if required), yielding results for the component itself and not just a certain material sample. This results in many applications, for example in quality assurance, structural health monitoring and the observation of material aging. The set-up for an ultrasonic inspection typically consists of a transducer that generates the ultrasound, the specimens through which the ultrasound is transmitted and a receiving transducer. The received signals are then analysed to obtain information about the specimens properties.

In this paper, plate-shaped specimens are examined. In addition to wide availability, they also have the advantage that transducers for excitation and detection of the acoustic wave can be placed freely on the plate's surface. Thereby, the distance that the wave has to travel through the specimen can be varied. Other experimental set-ups that use, for example, cylindrical specimens (Bause et al., 2015) are limited to a fixed distance per specimen. Some experiments on plates are conducted using piezoelectric transducers for both excitation and detection of the waves (Rogers, 1995, Vish- nuvardhan et al., 2007). This has the advantage of high sensitivity; however, the size of the active area of the transducers limits the detectable wavelength to a certain minimum. Also piezoceramic transducers typically have a limited bandwidth in both detecting and emitting acoustic waves. Focused, pulsed laser radiation can be used for a broadband creation of sonic waves, utilizing the photoacoustic effect (Bell, 1880, Hutchins et al., 1989). This allows for the excitation of many transmission modes at the same time. A multi-modal analysis is favourable, especially for orthotropic materials, as the components of the elastic moduli affect each mode differently and with different sensitivities (Vishnuvardhan et al., 2007). Past studies of guided acoustic waves in plateshaped specimens either relied on laser Doppler vibrometers (Hutchins et al., 1989, Bermes et al., 2007) or utilized transducers based on piezoelectric films such as polyvinylidene fluoride (Coufal et al., 1992) for the detection. Experiments with the piezofilm transducer used by Singer et al. (2014) yield no satisfactory results on polymers, as the sensitivity of the transducer is too low. The high cost factor of a laser Doppler vibrometer raises the question of a more affordable alternative technique. Because of this, a transducer based on a piezoceramic strip is developed, unifying a high detection bandwidth with high sensitivity. 
(a) Cross section

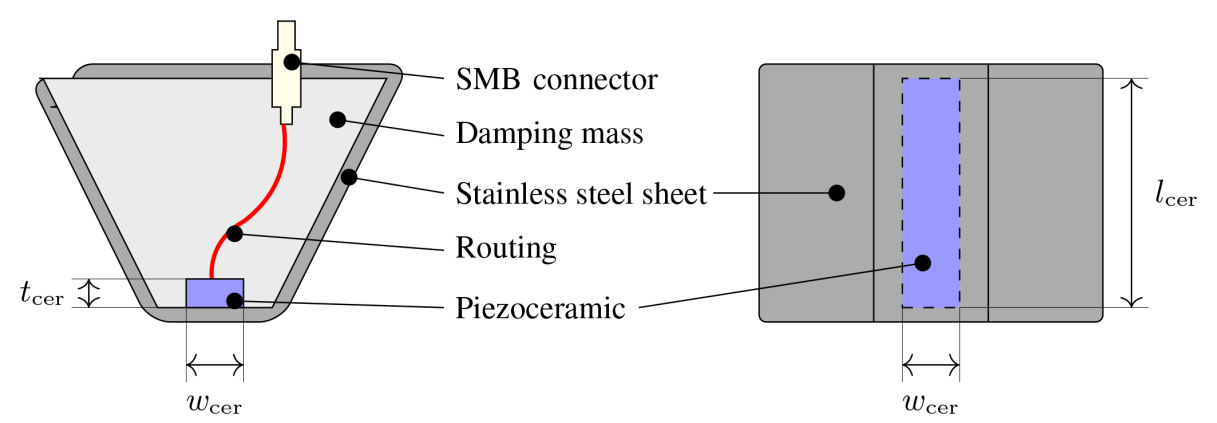

Figure 1. Cross section and bottom view of the purpose-build, piezoceramics-based transducer for the detection of plate waves.

The examined specimen (a polymer plate made of polyamide 6) is used to illustrate that the presented procedure can be applied to materials with high damping. Because many modes are being excited, the evaluation of a single measured signal proves difficult. Instead, the distance between excitation and detection of the ultrasound is varied. Using traditional ultrasonic transmitting transducers, this would be hard to realize with regards to reproducibility. The transducers would have to be moved and the acoustic coupling would change upon every step. In the presented measurement set-up, the distance can be varied easily and highly reproducibly by moving the focus of the laser radiation, using a linear actuator. The resulting data are then transformed from the spatial and temporal domain to frequency and wavenumber, resulting in a depiction similar to a dispersion diagram. This is done by applying a two-dimensional Fourier transform (Alleyne and Cawley, 1990) and alternatively via the linear prediction method (Grosh and Williams, 1993). This way, the dependence on absolute time and distance can be neglected, the data now only depend on the increment in space and time. Those increments can be specified with higher accuracy, as they are given by the measurement equipment (linear actuator and oscilloscope, respectively). The dispersion diagram generated by this procedure provides information on the modes being excited by the laser radiation and can be analysed further to yield information about the specimen's material properties. An inverse procedure is implemented by comparing the result of the linear prediction to simulated dispersion diagrams using an image-based approach.

\section{Realization of the piezoceramic transducer}

The presented transducer is a progression on the work shown in Claes et al. (2015). It features increased sensitivity and bandwidth, a better shielding against electromagnetic interference and no longer requires a conductive sheet to be placed between transducer and specimen. The increased sensitivity is especially required to yield satisfactory signals when examining polymeric specimens with high damping. Compared to transducers based on piezoelectric polymer films, as presented for example by Coufal et al. (1992), the piezoceramic-based transducer retains the drawback of a limited spatial detection ability, similar to the Nyquist criterion. For waves to be detected unattenuated, their wavelength has to be a least twice the width of the piezoceramic:

$\lambda>2 \cdot w_{\mathrm{cer}}$.

For the detectable angular wavenumber $k$ this results in an upper limit:

$k<\frac{2 \pi}{\lambda}=\frac{\pi}{w_{\text {cer }}}$.

For the presented transducer $\left(w_{\text {cer }}=1 \mathrm{~mm}\right)$, the limit results in $k<3142 \mathrm{rad} \mathrm{m}^{-1}$. However, measurements (Figs. 9, 10) show that the limit is not as critical as expected. Signals with an angular wavenumber larger than $3142 \mathrm{rad} \mathrm{m}^{-1}$ are clearly visible in the results.

The transducer is constructed using a cuboid PZT (lead zirconate titanate) piezoceramic (type PIC255, PI Ceramics $\mathrm{GmbH}$, Germany) with the dimensions $l_{\mathrm{cer}}=12 \mathrm{~mm}$, $w_{\mathrm{cer}}=1 \mathrm{~mm}$ and a thickness of $t_{\mathrm{cer}}=0.5 \mathrm{~mm}$. The piezoceramic is polarized in the direction of the thickness with the electrodes placed accordingly. Using epoxy resin mixed with silver particles, the piezoceramic is adhered to a $50 \mu \mathrm{m}$ thick stainless steel sheet. Adding the silver particles to the adhesive yields an electrically conductive coupling. A wire is connected to the back electrode of the piezoceramic using low-temperature solder paste to avoid depolarizing the piezoceramic. The stainless steel sheet is then folded into a trapezoid shape, as shown in Fig. 1. A hole is drilled through the sheet to mount a coaxial connector (here a SMB (SubMiniature version B) connector is used). Compared to transducers based on piezoelectric polymers, piezoceramics normally do not exhibit broadband sensitivity. Piezoceramics usually are sensitive mainly around their antiresonance frequency. For the piezoceramic to be used as a broadband sensor, the resonances need to be damped. The trapezoid shape is therefore 


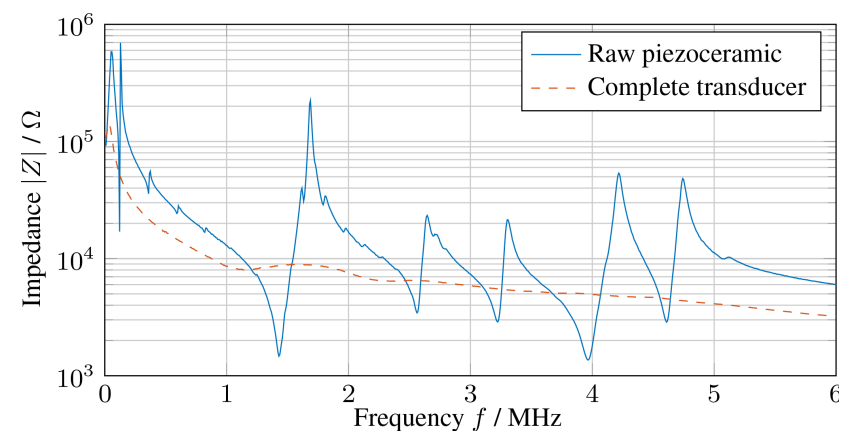

Figure 2. Absolute value of the ceramic's electrical impedance before and after embedding in the damping mass.

filled with a damping mass consisting of polyurethane mixed with tungsten carbide (Bause et al., 2013). The mixing ratio of tungsten carbide and polyurethane is $5 / 1$ (mass tungsten carbide / mass polyurethane) to yield an acoustic impedance of the damping mass of about 7 MRayl. In addition to damping the resonances of the piezoceramic, the damping mass stabilizes the transducer mechanically.

Figure 2 shows the absolute value of the electrical impedance of the raw piezoceramic (solid line) and the finished transducer (dashed line). The undamped piezoceramic shows distinct resonance-antiresonance pairs. A comparison with the impedance of the complete transducer shows that the resonances of the ceramic are almost completely damped. Only the resonance-antiresonance pairs at about 1.5 and $2.5 \mathrm{MHz}$ remain visible as a combination of minimum and maximum in the impedance plot. Judging from the frequency-dependent impedance, one can assume that the transducer exhibits a uniform sensitivity over the considered frequency band. The overall lower impedance can be attributed to a larger static capacity compared to the bare piezoceramic resulting from stray fields between the ceramic and the stainless steel sheet.

To further evaluate the transducer's detection capabilities, a broadband ultrasonic source is required. For this the photoacoustic effect can be utilized, similar to the experimental set-up described in the following section. Using a short ultraviolet radiation pulse to heat up the active area of the transducer, a signal that displays similarity to a step response is recorded. The radiation is emitted by a laser (MNL 103PD ${ }^{\text {High Power }}$, LTB Lasertechnik Berlin GmbH, Germany) with a pulse width of $3 \mathrm{~ns}$ (full width at half maximum) and pulse energy of $225 \mu \mathrm{J}$. As the laser radiation is not focused but evenly distributed on the transducer's active area, it can be assumed that no ablation takes place. The transducer's active area is heated up by the radiation abruptly. It is, however, assumed that it takes a significantly larger amount of time to cool down. Due to thermal expansion, a similar trend (a step followed by slow exponential decay) is expected for the displacement in the material. The acquired, normalized step response $h(t)$ is depicted in Fig. 3 (solid line). The recorded

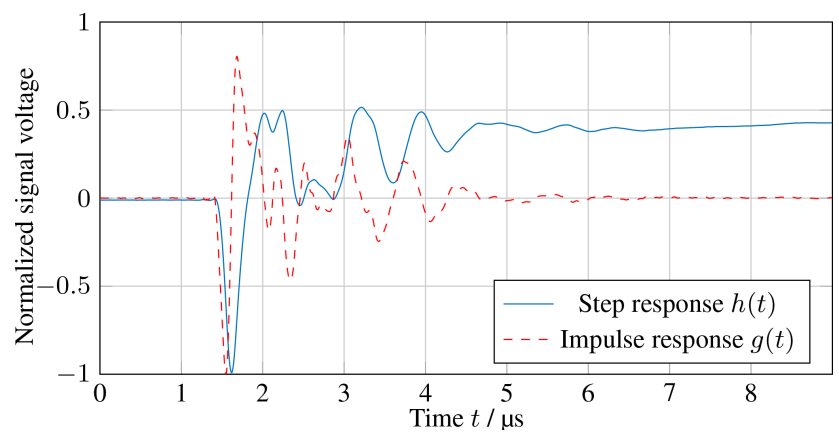

Figure 3. Step response and impulse response of the transducer. Recorded by heating up the transducer's active area with a short burst of laser radiation.

signal is similar to the step response of a damped oscillatory system. This supports the assumption that the laser excitation creates a step in the displacement of the material of the transducer's active area. No unique frequency is apparent, suggesting that multiple resonance frequencies of the piezoceramic are excited. The initial step is followed by an exponential decay with a time constant $\tau$ of $0.5 \mathrm{~ms}$ (not depicted). The decay may be caused by the discharge of the transducer's capacitance or result from the induced temperature gradient via the pyroelectric effect. As the decay is a low-frequency process, its influence on the analysis of the transducer's ultrasonic detection capabilities is negligible. Deriving the step response $h(t)$ yields the impulse response $g(t)$ :

$g(t)=\partial_{t} h(t)$.

The normalized impulse response is depicted in Fig. 3 as well (dashed line).

Assuming that the transducer is a linear and time-invariant system, its frequency response can be derived from the impulse response using the well-known relation

$G(\mathrm{j} \omega)=\mathcal{F}\{g(t)\}$,

where $\mathcal{F}$ is the Fourier transform operator. The absolute value of $G(\mathrm{j} \omega)$ is depicted in Fig. 4. Judging from the frequency response, signals with frequencies from 1 to $100 \mathrm{kHz}$ are detected with the same sensitivity. There is a slight decrease in sensitivity at $300 \mathrm{kHz}$ followed by four peaks between 1 and $4 \mathrm{MHz}$. The dip around $300 \mathrm{kHz}$ may be caused by an antiresonance frequency, which is visible in the impedance plot of the undamped piezoceramic. The first peak coincides with the remaining resonance frequency of the damped piezoceramic (Fig. 2, dashed line), while the second to fourth can be accounted to resonance frequencies of the undamped piezoceramic (Fig. 2, solid line). This indicates that effects of the resonance frequencies are present even if they are not visible in the impedance plot. At frequencies above $4 \mathrm{MHz}$ the frequency response drops sharply and becomes erratic. This suggest an upper limit in the sensors 


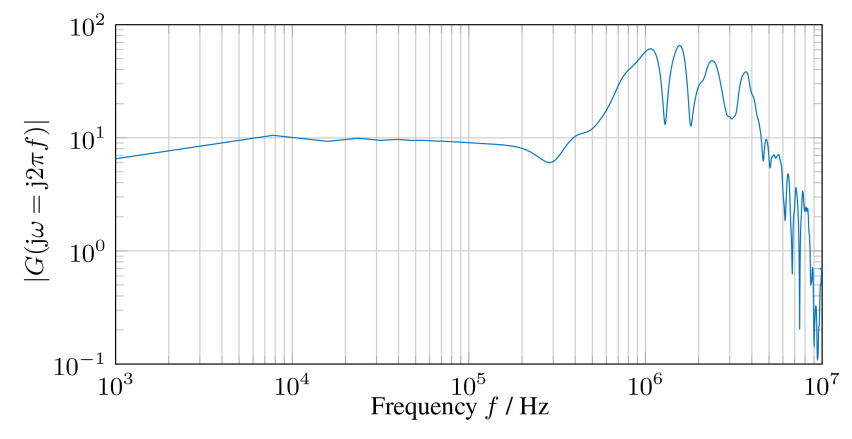

Figure 4. Absolute value of the frequency response of the transducer.

sensitivity at about $5 \mathrm{MHz}$. The erratic trend is due to an insufficient signal-to-noise ratio at higher frequencies. The frequency response, however, does suggest broadband detection capabilities from about $1 \mathrm{kHz}$ to $5 \mathrm{MHz}$. This is confirmed by empirical tests, which show that the sensor is capable of detecting sound in the audible range. The measurements presented below further show detected signals in the megahertz regime. If a simple model for the transducer is required, a high-order low pass with medium quality factor can be used. A more elaborate model would be a series of second-order low-passes with the resonance frequencies of the piezoceramic.

It should be noted that this particular method of estimating a transducer's frequency response only yields qualitative results. This is mainly due to the fact that the initial signal (the step) is not measured directly. Although the recorded signal resembles a step response, we cannot be sure if the excitation is step shaped. To evaluate this, a measurement of the displacement of the transducer's active area, using for example a vibrometer, is necessary. This would then enable a quantitative analysis of the transducer's detection capabilities. As the voltage of the recorded signals is sufficient and noise presents no problem, the described qualitative estimation of the frequency response is considered acceptable.

\section{Experimental set-up}

To examine the material properties of plate-shaped specimens, the experimental set-up shown schematically in Fig. 5 is used. The set-up of the measurement is similar to the ones presented for example by Schneider et al. (1997) and Coufal et al. (1992). To the authors' knowledge, all previous setups applied transducers based on polymeric piezoelectrics. Pulsed laser radiation (nitrogen laser MNL 103-PD ${ }^{\text {High Power }}$, LTB Lasertechnik Berlin GmbH, Germany) is focused on a line on the specimen's surface using a cylindrical lens. The radiation pulses locally heat up the specimen for a short time ( $3 \mathrm{~ns}$ full duration at half maximum), which results in the generation of acoustic waves (Bell, 1880). The excitation on a line creates acoustic waves with straight wavefronts. The

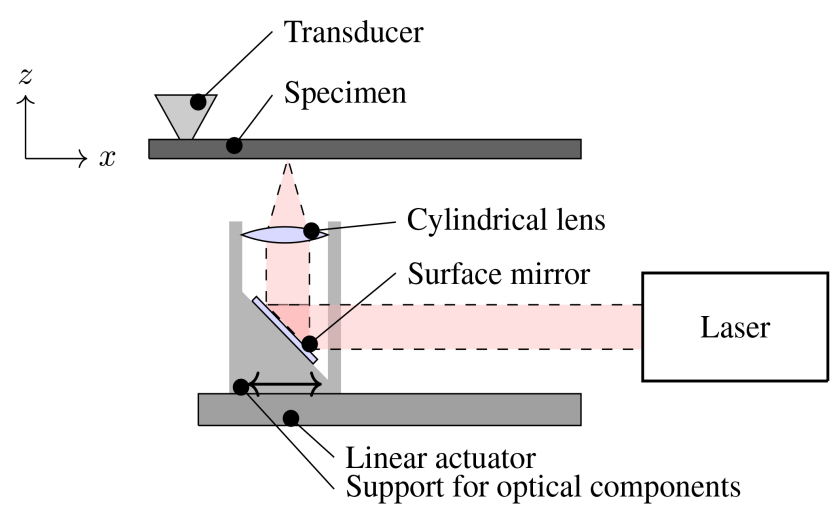

Figure 5. Experimental set-up for the determination of material properties utilizing the photoacoustic effect and the transducer shown in Fig. 1.

line also distributes the electromagnetic energy introduced to the specimen's surface to a larger area, so that no ablation of material occurs. This would otherwise conflict with the claim of non-destructiveness. Using a line of excitation also limits the distortion of the signal, which would be caused by examining circular waves using a line-shaped detector. The optical elements (the cylindrical lens and a front surface mirror) are mounted on a linear actuator (T-LSM, Zaber Technologies Inc., Canada) to adjust the distance between the line of excitation and detection of the acoustic waves. This enables one to pick up measurement signals with spatial resolution and can be used to observe the changes in the ultrasonic impulse's shape as it travels through the specimen. The transducer is placed on the surface of the specimen with the ceramic strip aligned in parallel to the line of excitation. A small amount of couplant (multi-range coupling paste ZGT, GE Measurement \& Control, USA) ensures the transmission of acoustic waves from the specimen to the transducer. Detecting the plate waves on one surface of the specimen while exciting them on the other allows for measurements very close to and directly above the line of excitation. A charge amplifier (HQA-15M-10T, FEMTO Messtechnik GmbH, Germany) is used to condition the signals for further analysis. Signals are recorded using a USB oscilloscope. Measurements and laser pulses are triggered simultaneously by a signal generator. This allows for repeated, identical measurements to improve the signal-to-noise ratio by forming the ensemble average of multiple signals.

\section{Measurements and analysis}

\subsection{Time domain}

For an estimation of the specimens longitudinal and transversal wave velocity, the following measurement, similar to the one proposed by Castagnede et al. (1991), is conducted: the focus line of the laser is moved from one side of the trans- 


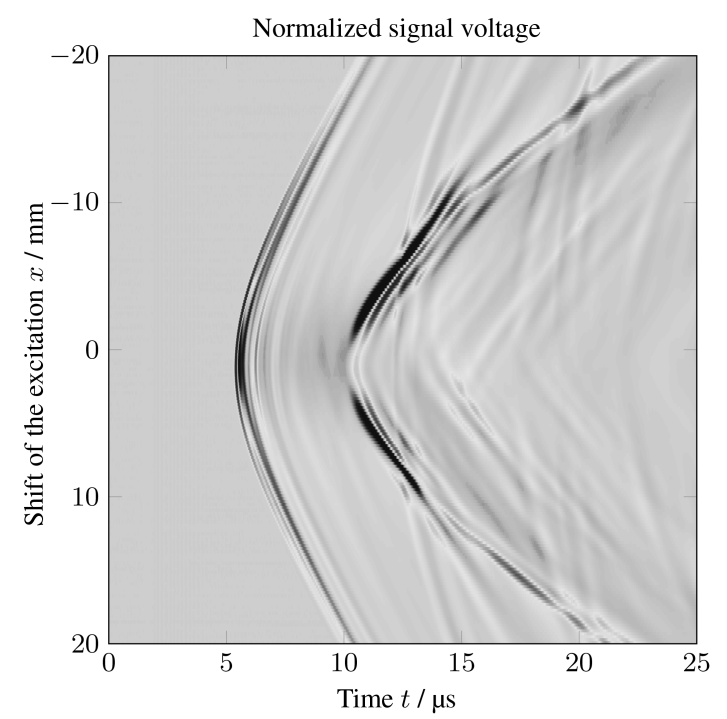

Figure 6. Measurement data (normalized signal voltage) with temporal and spatial resolution. Generated by moving the focus of the laser in equidistant steps below the transducer and recording a signal at every step. The specimen is a polyamide 6 plate, thickness is $8.8 \mathrm{~mm}$.

ducer to the other in equidistant steps while recording a signal at every step. The resulting data are arranged in a twodimensional matrix with spatial and temporal resolution as shown in Fig. 6. This matrix can be used to determine the position of the ultrasonic transducer relative to the starting position of the linear axis. If the focus of the laser is located directly below the transducer, the path the acoustic wave has to travel is the shortest. Therefore, the position at which the signal arrives earliest has to be the position of the transducer on the other side of the plate. For further measurements $x$ is set to 0 at this position.

Close to $x=0$, there are two distinct signal groups visible. One arrives at about $5 \mu$ s and a second at about $10 \mu$ s. These two signals can be assigned to a longitudinal and a transversal wave, respectively (Schröder and Scott Jr, 2001). This shows that close to the line of excitation, the observed behaviour is more similar to bulk waves than to plate waves. The propagation of waves as guided plate waves occurs farther from the excitation. It is to be noted that the signal for the transversal wave (the one arriving at about $10 \mu \mathrm{s}$ ) is weaker around $x=0$. This is in accordance with the results of Schröder and Scott Jr (2001), which also show a minimum in the transversal wave directly below the excitation. Both the longitudinal and transversal wave velocity can be estimated by dividing the thickness of the specimen by the delay at which both waves are detected by the transducer. The procedure applied is illustrated in Fig. 7. As it is difficult to determine the exact point of arrival of longitudinal and transversal wave, a method of estimation is required. Therefore, the envelope of the signal measured at $x=0$ is con-

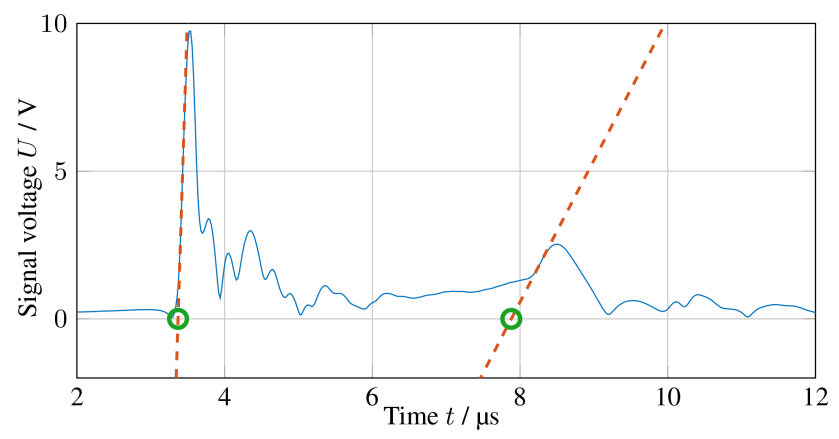

Figure 7. Envelope of the signal measured with the excitation located directly below the transducer (solid). With tangents (dashed) in the inflection points before the maxima to estimate the arrival of the longitudinal and transversal waves.

sidered (solid line in Fig. 7). There are two maxima corresponding with both wave velocities. To estimate the arrival of each respective wave, tangents are placed in the inflection points that occur before each maximum (dashed lines in Fig. 7). The point of intersection of the tangents and the $t$ axis is then used as an estimation for the time it takes the longitudinal and transversal waves to cross the specimen in thickness direction. With the thickness of the specimen $(8.8 \mathrm{~mm})$ the wave velocities can be estimated as $c_{1}=2611 \mathrm{~m} \mathrm{~s}^{-1}$ and $c_{\mathrm{t}}=1117 \mathrm{~m} \mathrm{~s}^{-1}$. This arbitrary method is only used to obtain rough estimates that will serve as initial values in the material characterization discussed later.

Figure 8 shows the results of a measurement similar to the one in Fig. 6. This time, the focus of the laser is moved away from the transducer, starting at the position determined to be $x=0$ in the prior measurement. This measurement allows for an observation of the forming of propagation modes in the plate. While one can observe distinct longitudinal and transversal waves while the distance between the laser's focus and the transducer is small, we can detect different propagation modes at larger distances. At a distance of $x=50 \mathrm{~mm}$ there are at least two different propagation modes visible. The first is detected at about $20 \mu \mathrm{s}$ and the second at about $50 \mu \mathrm{s}$. It is also visible that the faster mode is weaker and contains higher-frequency components than the slower, dominant signal group. One might assume that these signals may still be longitudinal and transversal bulk waves. However, the following analysis in the frequency domain shows that there are in fact many superimposed modes being excited by the laser.

\subsection{Frequency domain}

\subsubsection{Two-dimensional Fourier transform}

Alleyne and Cawley (1990) described a method to analyse waveguides by calculating a dispersion diagram from spatially and temporally resolved data. Applying a twodimensional Fourier transform to such data yields a depiction 


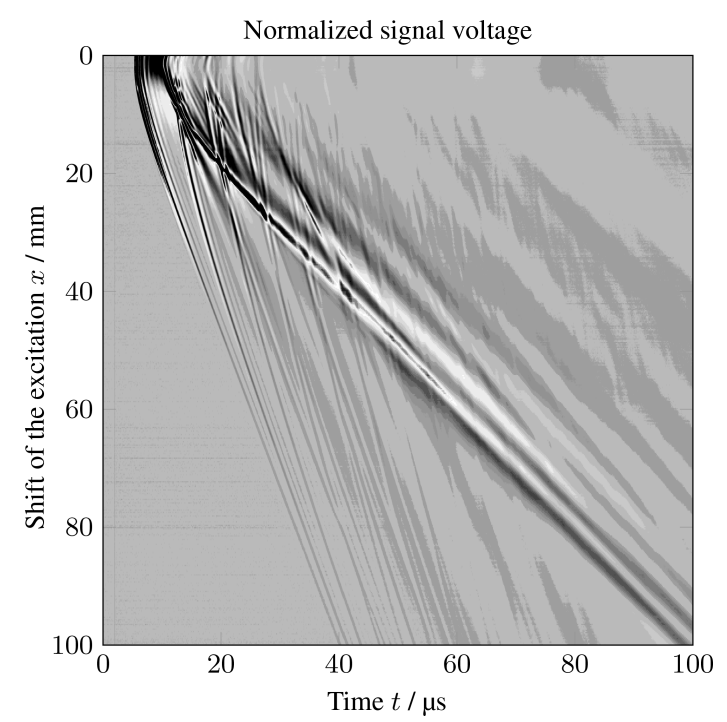

Figure 8. Measurement data (normalized signal voltage) with temporal and spatial resolution. Generated by moving the focus of the laser away from the transducer in equidistant steps. The specimen is a polyamide 6 plate, thickness is $8.8 \mathrm{~mm}$.

with wavenumber and frequency resolution:

$\mathcal{F}_{2-\mathrm{D}}\{u(x, t)\}=U(k, \omega)$.

Given that signal components from every mode are present in the measured signals, the depiction shows ridges where curves are expected in a normal dispersion diagram. Figure 9 shows the result of a two-dimensional FFT (fast Fourier transform) applied to the measured data shown in Fig. 8. Compared to the same measurement conducted on a metallic specimen (for example copper, see Fig. 10) the different ridges are not as easily distinguishable. This is primarily due to the specimen's properties. The low-longitudinal and transversal wave velocities in polymers combined with the ticker plate $(8.8$ vs. $1.53 \mathrm{~mm})$ yield a substantially larger number of modes that are capable of propagation. The copper plate, however, shows several ridges that are easily distinguishable. A characterization of the copper specimen was presented in Claes et al. (2015), although the measurement was conducted using a predecessor of the transducer presented above. Figure 10 also demonstrates the broadband detection abilities of the presented transducer. Ridges corresponding to propagation modes up to $10 \mathrm{MHz}$ are visible. Due to the low-pass characteristic of the transducer (see Fig. 4), the ridges become less visible at higher frequencies. The limited wavenumber resolution given by the transducer's width (see Eq. 2) is not visible. However, a limit is given due to the spatial sampling given by the increment with which the linear actuator and thereby the excitation is moved.

To allow for a possible comparison with simulation results for an inverse procedure, the ridges of the depiction need to be detected automatically. For the polymeric specimen, this

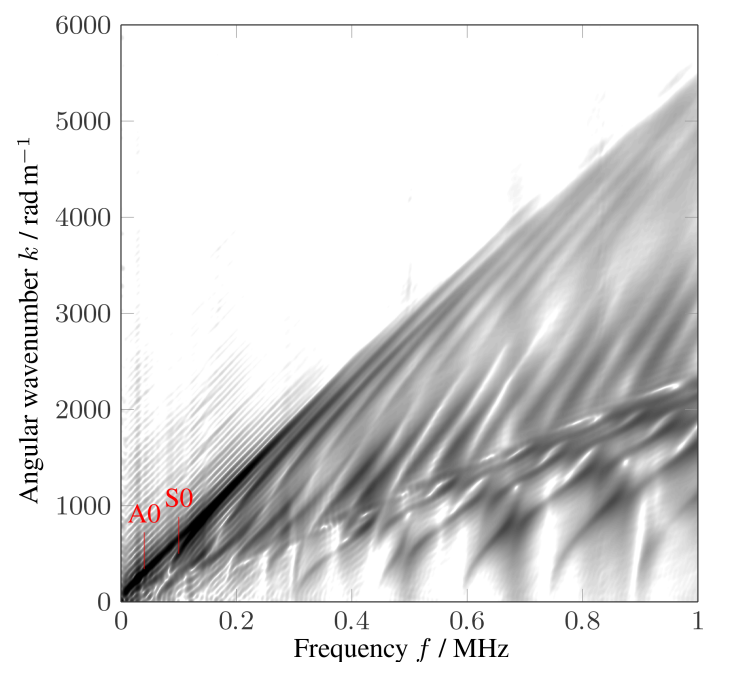

Figure 9. Dispersion map of a polymer plate (polyamide 6 , thickness $8.8 \mathrm{~mm}$ ). Generated by applying the two-dimensional FFT to spatially and temporally resolved data.

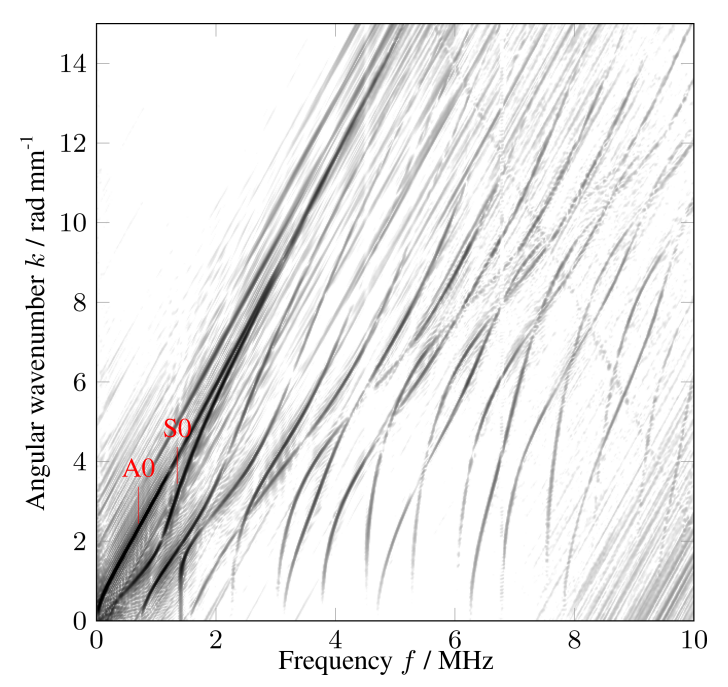

Figure 10. Dispersion map of a copper plate (thickness $1.53 \mathrm{~mm}$ ). Generated by applying the two-dimensional FFT to spatially and temporally resolved data.

proves to be problematic, as the ridges are too close to each other and too thick to be distinguished. An alternative is proposed in the following section.

\subsubsection{Linear prediction method}

Grosh and Williams (1993) and Plona et al. (1992) developed an approach based on linear prediction that allows for a direct computation of the dominant angular wavenumbers for a given frequency. The procedure is illustrated in Fig. 11. First, the matrix shown in Fig. 8 is Fourier transformed only in the time direction yielding a depiction with space and frequency 


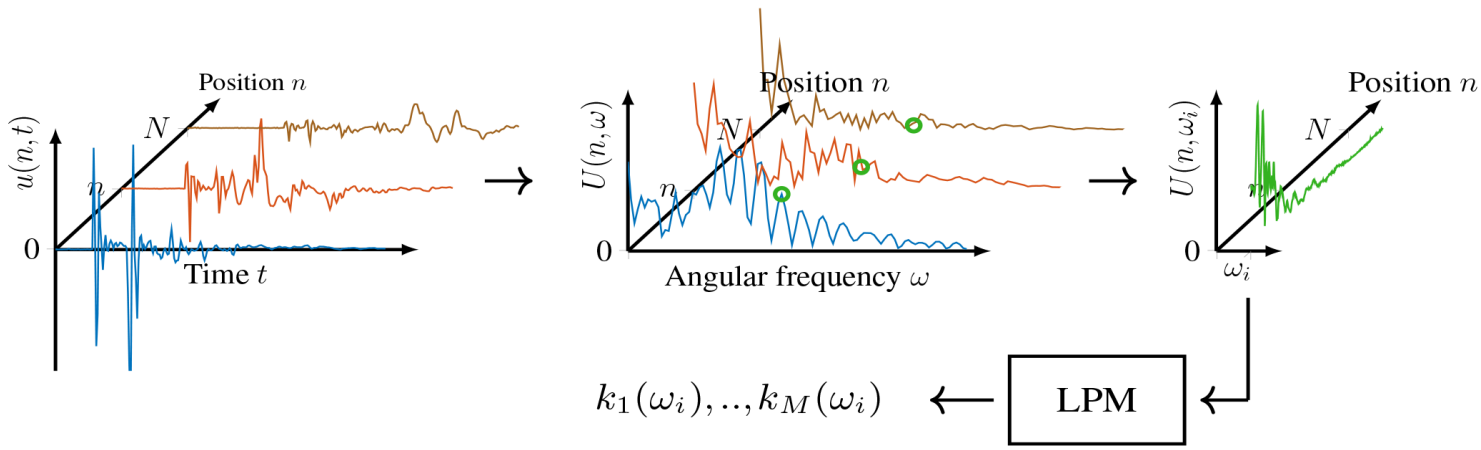

Figure 11. Concept of the application of the linear prediction method for the calculation of angular wavenumbers $k_{m}$ for a each angular frequency $\omega_{i}$.

resolution:

$\mathcal{F}\{u(x, t)\}=U(x, \omega)$.

Given that there are discrete measurement positions, $x$ is replaced by $n \cdot \Delta x, n \in \mathbb{N}$. As Fig. 11 shows, the matrix can be considered as $N$ spectra (one for each measurement position). Assuming a fixed $\omega_{i}$, a column of the matrix $U\left(n, \omega_{i}\right)$ is expressed as a superposition of $M$ complex oscillations:

$U\left(n, \omega_{i}\right)=\sum_{m=1}^{M} C_{m}\left(\omega_{i}\right) \cdot\left(\mathrm{e}^{-\mathrm{j} k_{m} n \Delta x}\right)=\sum_{m=1}^{M} C_{m}\left(\omega_{i}\right) \cdot \mu_{m}^{n}$,

where $C_{m}\left(\omega_{i}\right) \in \mathbb{C}$ and $k_{m} \in \mathbb{R}$. Applying the linear prediction method, a matrix is formed to estimate the values $U\left(L+1, \omega_{i}\right) \ldots U\left(N, \omega_{i}\right)$, from the preceding $L$ values using the weighting factors $g_{1} \ldots g_{L}$. For better readability, the constant parameter $\omega_{i}$ is omitted in the following equations.

$\left[\begin{array}{cccc}U(L) & U(L-1) & \ldots & U(1) \\ U(L+1) & U(L) & \ldots & U(2) \\ \vdots & \vdots & & \vdots \\ U(N-1) & U(N-2) & \ldots & U(N-L)\end{array}\right]\left[\begin{array}{c}g_{1} \\ g_{2} \\ \vdots \\ g_{L}\end{array}\right]=\left[\begin{array}{c}U(L+1) \\ U(L+2) \\ \vdots \\ U(N)\end{array}\right]$.

In short, this can be expressed as

$\mathrm{A} g=f$.

The weighting vector $\boldsymbol{g}$ is estimated using the pseudoinverse of $\mathbf{A}, \mathbf{A}^{+}$:

$g=\mathbf{A}^{+} f$.

Inserting Eq. (7) into the lines of the matrix A yields the following expression:

$$
\begin{aligned}
& \sum_{m=1}^{M} C_{m}\left(\mu_{m}\right)^{L+1}-\sum_{m=1}^{M} C_{m}\left(\mu_{m}\right)^{L} g_{1}- \\
& \sum_{m=1}^{M} C_{m}\left(\mu_{m}\right)^{L-1} g_{2}-\ldots-\sum_{m=1}^{M} C_{m}\left(\mu_{m}\right) \cdot g_{L}=0 .
\end{aligned}
$$

Factorizing results in

$$
\sum_{m=1}^{M} C_{m} \mu_{m}\left(\mu_{m}^{L}-\mu_{m}^{L-1} g_{1}-\mu_{m}^{L-2} g_{2}-\ldots-g_{L}\right)=0 .
$$

To satisfy this equation, the expression in brackets has to be equal to zero. As the weighting factors $g_{1} \ldots g_{L}$ are given by Eq. (10), this equation can be solved for $\mu_{m}$, which yields $L$ different complex roots. These roots are contained within the unit circle and represent the different oscillations present in the signal. Their absolute value provides a measure for their significance. For further analysis, the $P$ complex roots that have the largest absolute value and are thereby closest to the unit circle are selected. Given that $\mu_{m}=\mathrm{e}^{-\mathrm{j} k_{m} \Delta x}$, the respective angular wavenumber can be calculated as

$k_{m}=\frac{\arg \left(\mu_{m}\right)}{\Delta x}$.

The linear prediction method, as conducted in this paper, has two parameters. The first is $L$, the number of weighting factors and thereby the number of wavenumbers $k_{m}$ to be calculated. In practice, setting $L$ to one-third of the measurement positions yields good results. The second parameter is the number of complex roots closest to the unit circle $P$. They represent the $P$ wavenumbers that are calculated per frequency step. If $P$ is too high, there might be a lot of wavenumbers obtained that are not part of a mode, but result from noise or other interferences. In waveguides the number of propagation modes, and thereby the number of detectable wavenumbers, increases with the frequency. As a means to resolve this issue, instead of choosing a fixed $P$ for all frequencies, a lower bound for the distance of the complex roots $\mu_{m}$ to the unit circle is set. All roots that exceed this bound are considered to represent dominant wavenumbers for the respective frequency.

The result can be printed as a point cloud, as shown in Fig. 12. It is similar to a dispersion diagram, however there are some modes that are not detected, some curves are interrupted and some points are implausible (e.g. those that are above the A0-Mode). To enhance the result for further analysis, it is checked if the resulting data points form a curve by checking their distance to each other. If a detected curve contains fewer than a given number of data points (e.g. $<20)$, it 


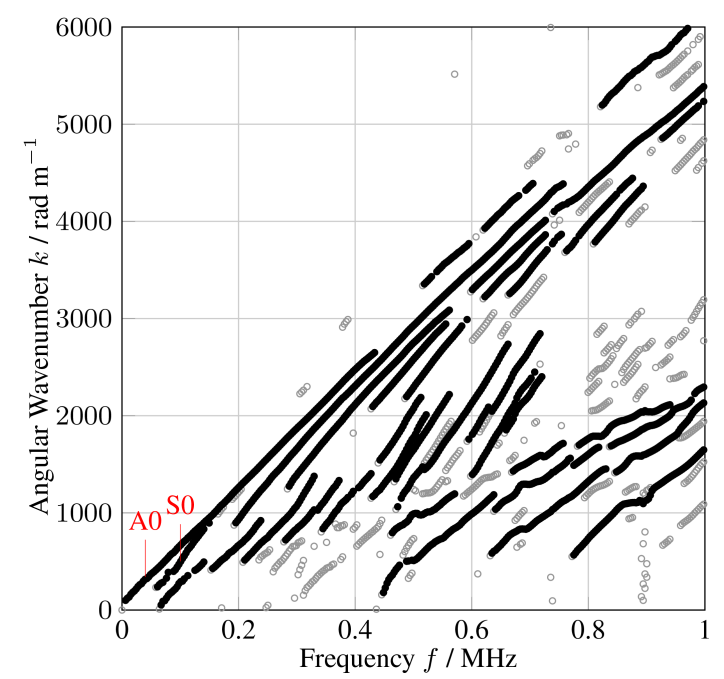

Figure 12. Result of the linear prediction method for dispersion analysis applied to data measured on a polymer plate (polyamide 6, thickness $8.8 \mathrm{~mm}$ ). Grey points are omitted.

is discarded. In Fig. 12, the points that are omitted are printed grey.

\section{Material characterization}

The results of the linear prediction method can be used to characterize the material's properties by applying an inverse approach. To realize this approach, a forward model, a cost function and initial values for the model parameters are required.

Lamb's characteristic equations are used as the forward model, with the transversal and longitudinal wave velocities $\left(c_{\mathrm{t}}\right.$ and $\left.c_{1}\right)$ and the thickness of the plate $d$ as parameters (Lamb, 1917):

$\frac{\tan (\beta d / 2)}{\tan (\alpha d / 2)}=-\left(\frac{4 \alpha \beta k^{2}}{\left(k^{2}-\beta^{2}\right)^{2}}\right)^{ \pm 1}$

where

$\alpha^{2}=\frac{\omega^{2}}{c_{1}^{2}}-k^{2} \quad$ and $\quad \beta^{2}=\frac{\omega^{2}}{c_{\mathrm{t}}^{2}}-k^{2}$.

Based on them, a dispersion diagram is to be simulated and compared to the results of the linear prediction method. As two parameters of the simulation (longitudinal and transversal wave velocity) are varied to match the measurement result, the dispersion diagram has to be computed a number of times. Therefore, a method that allows for a fast computation of the dispersion diagrams is required. In this paper, the dispersion diagrams are created by numerically solving Lamb's characteristic equations using the MATLAB ${ }^{\circledR}$ function ezplot. This function applies a root-finding algorithm and allows for the computation of a dispersion diagram in

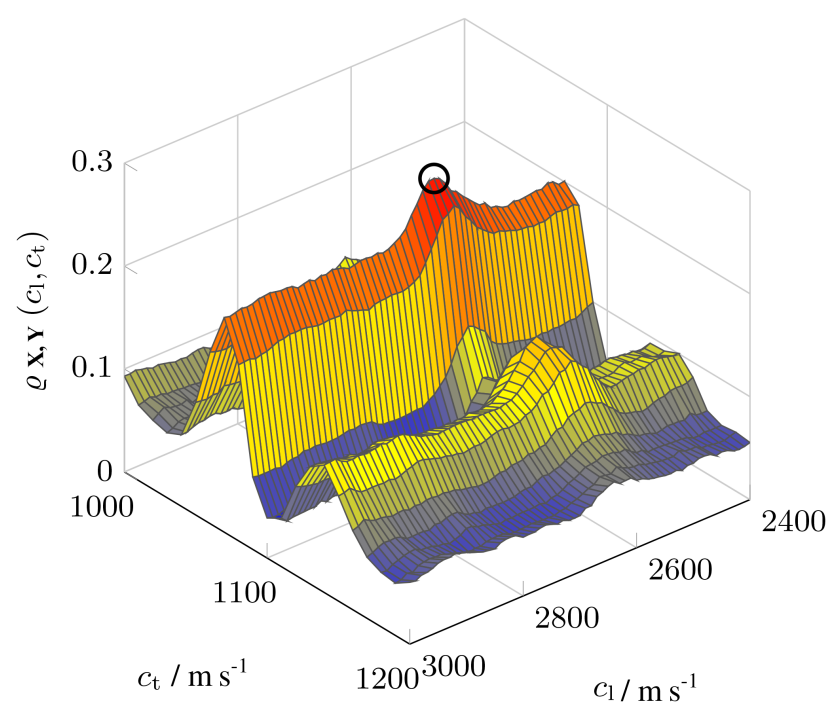

Figure 13. Correlation coefficient $\varrho \mathbf{X}, \mathbf{Y}\left(c_{1}, c_{\mathrm{t}}\right)$ of the measured and simulated dispersion diagram. Maximum at $c_{1}=2630 \mathrm{~m} \mathrm{~s}^{-1}$ and $c_{\mathrm{t}}=1070 \mathrm{~m} \mathrm{~s}^{-1}$.

a few seconds. While the resulting dispersion diagram is incomplete and shows gaps in some modes, it suffices for a comparison with the equally incomplete result of the linear prediction method.

A cost function is required for an automatic and quantifiable comparison of measurement and simulation. The setup of an appropriate cost function proves challenging as two plots with multiple incomplete lines consisting of discrete points are to be compared. This issue is solved by applying an image-based approach and calculating the correlation coefficient. To compare them, both dispersion diagrams are converted to two-dimensional matrices that contain ones where lines are found in the respective diagram and zeros elsewhere. These matrices are regarded as images in the following steps, where $\mathbf{X}$ is the image generated from the result of the linear prediction method and $\mathbf{Y}\left(c_{1}, c_{\mathrm{t}}, d\right)$ is the image generated from the simulation result. To compare $\mathbf{X}$ and $\mathbf{Y}\left(c_{1}, c_{\mathrm{t}}, d\right)$, the correlation coefficient of the two images is calculated. Comparing these two images directly, however, yields no usable results from the correlation coefficient, as the lines in the generated images are too sharp. Only modes with a pixel-perfect match would contribute to the correlation coefficient $\varrho \mathbf{X}, \mathbf{Y}$ in this case. To remedy this, Gaussian blur is added to both images to allow for the correlation coefficient to yield results if the images are similar but not identical. Using initial values for longitudinal and transversal wave velocities obtained from the measurement shown in Fig. 6, both parameters are swept. The third parameter of Lamb's characteristic equations, the thickness of the plate $(d=8.8 \mathrm{~mm})$, is measured directly and kept constant. Figure 13 shows the correlation coefficient $\varrho_{\mathbf{X}, \mathbf{Y}}\left(c_{1}, c_{\mathrm{t}}\right)$ of the two blurred images as a function of the longitudinal and transversal wave veloc- 


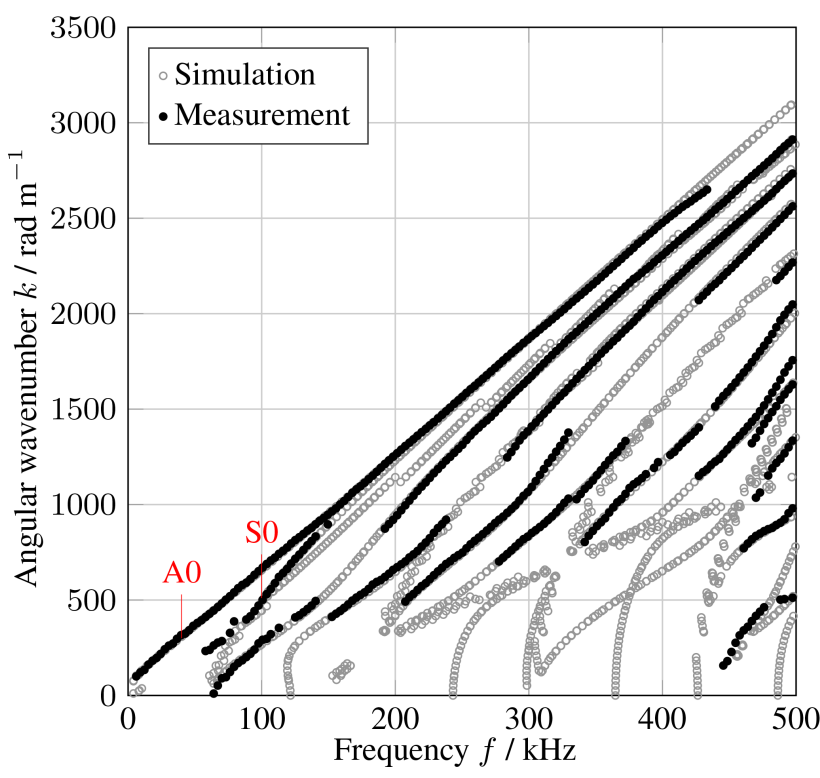

Figure 14. Comparison of simulated and measured dispersion diagram.

ity parameters $\left(c_{1}\right.$ and $\left.c_{\mathrm{t}}\right)$ of the simulation. There are orthogonal ridges visible indicating that a matched transversal and a mismatched longitudinal wave velocity (and vice versa) are registered by the correlation coefficient. The point where those ridges intersect yields the largest correlation coefficient and thereby an approximation for the materials parameters. These approximation are

$$
\begin{aligned}
& -c_{1}=2630 \mathrm{~m} \mathrm{~s}^{-1} \\
& -c_{\mathrm{t}}=1070 \mathrm{~m} \mathrm{~s}^{-1}
\end{aligned}
$$

A comparison between the simulated dispersion diagram and the result of linear prediction method is shown in Fig. 14. Even if both dispersion diagrams are incomplete, the different modes match well. The longitudinal and transversal wave velocities determined via ultrasound in other works (for example by Arnold and Guenther, 1966 and Asay and Guenther, 1967) are slightly higher, which may be due to material variation or the higher frequency used. Measuring the density $\rho$ of the specimen $\left(1142 \mathrm{~kg} \mathrm{~m}^{-3}\right)$ and using the determined wave velocities, other material parameters can be calculated (Mechel et al., 2013). These are for example Young's modulus $E$, Poisson's ratio $v$ and the shear modulus $G$ :

$$
\begin{aligned}
& -E=3.66 \mathrm{GPa} \\
& -v=0.40 \\
& -G=1.31 \mathrm{GPa}
\end{aligned}
$$

Compared to the results of tensile testing in the literature (Martienssen and Warlimont, 2006), Young's modulus $E$ deviates upwards. This is to be expected, as aforementioned tests yielded results for the quasi-static case. Polymers tend to stiffen when subjected to higher frequencies, which is represented by an increased Young's modulus. The estimated shear modulus and Poisson's ratio are in good agreement with literature data (Martienssen and Warlimont, 2006). As all parameters of the forward model (Lamb's characteristic equation) are considered frequency independent and the results are matched over a broad frequency range, the resulting estimations for the material parameters can be interpreted as effective quantities for the examined frequency range. To consider frequency dependencies in the material's parameters (for example the frequency dependence of Young's modulus) a more complex forward model needs to be applied. In addition to the frequency dependence of the examined parameters, further material properties that are common in polymers, such as damping and anisotropy, can be added to the model for a more accurate representation of the specimen.

\section{Summary and Outlook}

Using laser-generated, broadband ultrasonic plate waves, the material properties of a polymer specimen (polyamide 6) are determined. This is enabled by designing a broadband, piezoelectric-based ultrasonic transducer. Even if the results of the characterization of the transducer suggest low-pass behaviour with a cut-off frequency of about $5 \mathrm{MHz}$, modes are detectable at higher frequencies in metal specimens. They however become less pronounced as the frequency increases. To further expand the bandwidth of the transducer, an even smaller piezoceramic with higher resonance frequencies could be applied, although this would lead to a lower overall sensitivity. To facilitate the distinction of different modes in the dispersion map, the examination of thinner specimens is advised. This is particularly important when using polymer samples with low sound velocities. The examined material (polyamide 6) represents a simple isotropic material example. For this, the model using Lamb's characteristic equations suffices. The measurement of material properties of more complex samples, for example inhomogeneous, multi-layered or anisotropic specimens requires a more complex forward model. For example, a semi-analytical finiteelement (SAFE) model can be used (Aalami, 1973; Lagasse, 1973). More complex material models require more material parameters. For example, a transversely isotropic material has five independent parameters. Therefore, a simple sweep of the parameters to match simulation and measured dispersion map would no longer be feasible. Instead, a gradientbased optimization can be applied. 
Acknowledgements. The authors would like to thank the German Research Foundation (DFG) for financial support of the research projects HE 2897/3-1 (determination of acoustic material properties) and HE 2897/6-1 (evaluation of hydrothermal aging of continuous-fiber reinforced thermoplastics and development of an ultrasonic measurement system for non-destructive characterization of the state of aging for structural health monitoring and remaining life-time predictions).

Edited by: A. Schütze

Reviewed by: three anonymous referees

\section{References}

Aalami, B.: Waves in prismatic guides of arbitrary cross section, J. Appl. Mech., 40, 1067-1072, 1973.

Alleyne, D. and Cawley, P.: A 2-dimensional Fourier transform method for the quantitative measurement of Lamb modes, Ultrason., 2, 1143-1146, 1990.

Arnold, N. D. and Guenther, A. H.: Experimental determination of ultrasonic wave velocities in plastics as functions of temperature. I. Common plastics and selected nose-cone materials, J. Appl. Polym. Sci., 10, 731-743, doi:10.1002/app.1966.070100506, 1966.

Asay, J. R. and Guenther, A. H.: Experimental determination of ultrasonic wave velocities in plastics as functions of temperature. IV. Shear velocities in common plastics, J. Appl. Polym. Sci., 11, 1087-1100, doi:10.1002/app.1967.070110708, 1967.

Bause, F., Rautenberg, J., and Henning, B.: Design, modeling and identification of an ultrasonic composite transducer for target impedance independent short pulse generation, 16. Internationaler Kongress für Sensoren und Messtechnik (Sensor 2013), 14-16 May 2013, Nuremberg, Germany, 2013.

Bause, F., Gravenkamp, H., Rautenberg, J., and Henning, B.: Transient modeling of ultrasonic guided waves in circular viscoelastic waveguides for inverse material characterization, Meas. Sci. Technol., 26, 2015.

Bell, A. G.: Upon the production and reproduction of sound by light, Am. J. Sci., 20, 305-324, 1880.

Bermes, C., Kim, J.-Y., Qu, J., and Jacobs, L. J.: Experimental characterization of material nonlinearity using Lamb waves, Appl. Phys. Lett., 90, 021901, doi:10.1063/1.2431467, 2007.

Castagnede, B., Kim, K. Y., Sachse, W., and Thompson, M. O.: Determination of the elastic constants of anisotropic materials using laser-generated ultrasonic signals, J. Appl. Phys., 70, 150-157, doi:10.1063/1.350303, 1991.
Claes, L., Bause, F., Rautenberg, J., and Henning, B.: Detection of ultrasonic plate waves using ceramic strip transducers, in: Sensor Conferences Nuremberg 19-21 May 2015, Nuremberg, Germany, 2015.

Coufal, H., Grygier, R., Hess, P., and Neubrand, A.: Broadband detection of laser-excited surface acoustic waves by a novel transducer employing ferroelectric polymers, J. Acoust. Soc. Am., 92, 2980-2983, 1992.

Grosh, K. and Williams, E. G.: Complex wave-number decomposition of structural vibrations, J. Acoust. Soc. Am., 93, 836-848, 1993.

Hutchins, D. A., Lundgren, K., and Palmer, S. B.: A laser study of transient Lamb waves in thin materials, J. Acoust. Soc. Am., 85, 1441-1448, doi:10.1121/1.397981, 1989.

Lagasse, P.: Higher-order finite-element analysis of topographic guides supporting elastic surface waves, J. Acoust. Soc. Am., 53, 1116-1122, 1973.

Lamb, H.: On waves in an elastic plate, P. R. Soc. Lond. A-Conta., 114-128, 1917.

Martienssen, W. and Warlimont, H.: Springer Handbook of Condensed Matter and Materials Data, Springer, Berlin Heidelberg, Germany, 2006.

Mechel, F., Munjal, M., Vorländer, M., Költzsch, P., Ochmann, M., Cummings, A., Maysenhölder, W., Arnold, W., and Rudenko, O.: Formulas of Acoustics, Springer, Berlin Heidelberg, Germany, 2013.

Plona, T. J., Sinha, B. K., Kostek, S., and Chang, S.-K.: Axisymmetric wave propagation in fluid-loaded cylindrical shells. II: Theory versus experiment, J. Acoust. Soc. Am., 92, 1144-1155, 1992.

Rogers, W.: Elastic property measurement using RayleighLamb waves, Res. Nondestruct. Eval., 6, 185-208, doi:10.1007/BF01606381, 1995.

Schneider, D., Schwarz, T., Scheibe, H.-J., and Panzner, M.: Nondestructive evaluation of diamond and diamond-like carbon films by laser induced surface acoustic waves, Thin Solid Films, 295, 107-116, 1997.

Schröder, C. T. and Scott Jr, W. R.: On the complex conjugate roots of the Rayleigh equation: The leaky surface wave, J. Acoust. Soc. Am., 110, 2867-2877, 2001.

Singer, F., Meier, C., and Lindner, G.: Laser-Acoustic Measurement of Anisotropic Material Parameters, in: Proceedings of Sensors and Measuring Systems 2014; 17. ITG/GMA Symposium, 1-6, 2014.

Vishnuvardhan, J., Krishnamurthy, C. V., and Balasubramaniam, K.: Genetic algorithm based reconstruction of the elastic moduli of orthotropic plates using an ultrasonic guided wave singletransmitter-multiple-receiver SHM array, VTT Symp., 16, 16391650, 2007. 